\title{
Crack Reduction during Drying Process by Using Surfactant
}

\author{
Sandra Guzlena ${ }^{1}$, Gita Sakale ${ }^{1}$, Sergejs Certoks ${ }^{2}$ and Arnita Spule ${ }^{1}$ \\ ${ }^{1}$ Institute of Technical Physics, Faculty of Material science and Applied Chemistry, Riga Technical University, Paula Valdena 3, LV- \\ 1048, Riga, Latvia \\ ${ }^{2}$ LODE SIA, Lode Street 1, Priekuli country, Latvia
}

\begin{abstract}
In this research clay brick samples were made from illitic clays. Surfactant was added to clay mass during formation process to reduce crack generation during drying process. Surfactant amount was changed below critical micelle concentration (CMC). Nonionic surfactant Triton X-100 was used. Samples were prepared using extruder. Clay mass samples were analysed by granulometry, surfactant by contact angle and surface tension measurements and brick samples by microscope to determine crack amount. Changing surfactant amount closer to CMC is possible to reduce crack amount in sample during drying stage.
\end{abstract}

\section{Introduction}

Clay products have been used as building material already from ancient times, but it is still popular material in current building solutions. Nevertheless, there are still challenges to improve brick making technology, as an example, to decrease drying time, by using higher temperatures or lower moisture. But that can result in brick cracking. Brick cracking can cause mechanical strength decrease and big amount of defective bricks [1]. There are different ways to avoid this problem - glass fibre addition to clay mass [2], submicron particle addition (PVA submicron particles), temperature and moisture precise regulation during drying and firing process [3], and also addition of surfactants during material mixing before brick forming process [1], [4], [5].

Drying process is often one of the most complex operations in a manufacturing process. Drying does not only refer to the removal as much as possible moisture but also to the protection of the physical structure and appearance as an important part of the drying process [6].

Drying process can be split in three parts. At the beginning of stage $I$, the wet bricks and tiles have a moisture content $20-30 \%$. In this stage heat is added. When product heats up until it reaches a certain temperature. Moisture diffuses by capillary action and first evaporates from the region near the solid surface. As some of the surface moisture evaporate, more moisture is transported from interior of the solid to its surface. For optimal drying, this process should be done slowly, because if the heat transfer takes place quickly, it becomes impossible for the moisture inside to escape and because of this some cracking may occur. At the second stage moisture content is crucial due to the water level in the clay reaching the critical point. The critical brick moisture content is an important parameter for determining the coefficient of sensitivity of clays to drying. It means the moisture of a solid material must be determined experimentally. At the third stage, the drying rate can be increased by providing the maximum amount of heat to reach the minimum moisture content (approximately 3-10\%). Sometimes the damage done during drying process can be observed only after firing [6].

Mostly cracks appear during first part of drying process, which can be explained with tension between dry brick surface and wet brick core. This is due to nonlinear moisture and/or temperature changes in material. If surfactants are used tension between surface tension and material pour walls can be controlled, to improve moisture transport from brick core to surface [1]. Positive influence is also on clay particles to achieve maximal electrokinetically potential increase and wetting. This influences in good way electrostatic rejection between the particles by changing layered structure in fibrous state with high surface roughness [7], [8]. Clay surface properties changes with added surfactant amount. As Kowalski et. al. [1] have investigated that if dedocilsulfate and fluoric surfactants are used at very low concentrations $(0,001 \%)$ surfactant must have been used to reduce crack formation. Clays with tendency to swell, which can cause cracking in contact with amido-amin bas cationic surfactants decrease this risk and reduce cracking [4].

Triton X-100 surfactant which was used in this study was chosen, due to M. J. Sánchez-Martín et.al. [9] ilitic clays have better adsorption to surface than octadecyltrimethylammonium bromide (ODTMA) and sodium dodecyl sulphate (SDS). Most of the previous investigations restrained the concentration range below 
critical micelle concentration (CMC) or near $\mathrm{CMC}$, for which the maximum of adsorption was reached [1], [10]. $\mathrm{CMC}$ is concentrations where molecules of surfactants arrange into micelles and all additional surfactants added to the system go to micelles:

- Before reaching the $\mathrm{CMC}$, the surface tension changes strongly with the concentration of the surfactant.

- After reaching the $\mathrm{CMC}$, the surface tension remains relatively constant or changes with a lower slope.

- For the improvement of moisture transport the surfactants concentration should be below CMC [1].

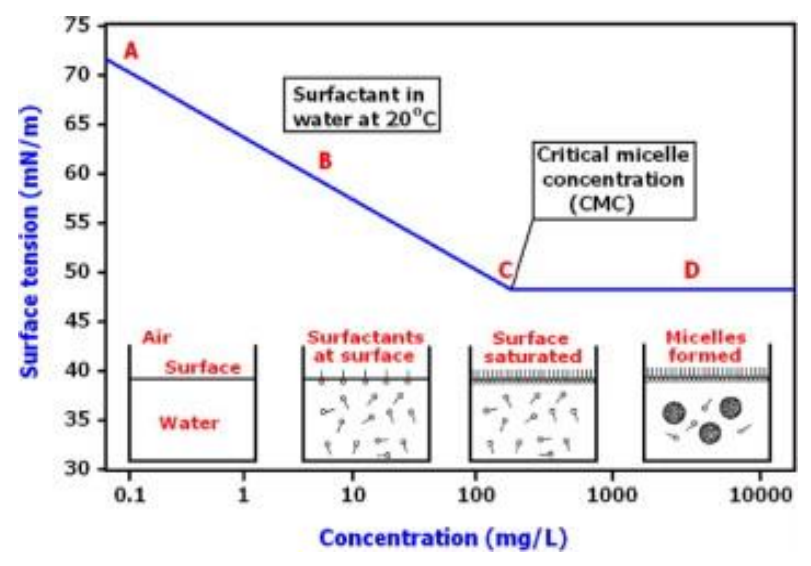

Figure 1. Micelle formation process [11].

Gluck (2010) study revealed that the CMC, an important property of surfactants, does not act as a threshold in the soil behaviour. If it did, soil properties would not change for concentrations higher than the CMC. But, as shown from experiments, increasing concentrations above the $\mathrm{CMC}$ had an effect on plasticity limit and particle compaction. [5]. So, in our research we use concentrations above CMC concentration.

\section{Materials and methods}

To form bricks four types of components were used grey, red and composite clay and sand from the biggest quarry in Latvia (Figure 2). Clay samples were analysed and discussed in our previous work [12]. Using raw material from quarry without additives considering only to grain size distribution requirements most appropriate is composite clay due to its balance between all fractions. To use grey and red clay, mixes must be stabilized with sand size particles to avoid unnecessary shrinkage during firing. Sand found on quarry would be suitable stabilizing additive for plastic clay. Illite and Kaolinite are the only clay minerals found in the samples. Suitable brick making material should have suitable proportion of illite, kaolinite and quartz. Illite could provide plasticity, kaolinite promotes fired hardness and sand grains act as a stabilizer and filler material.

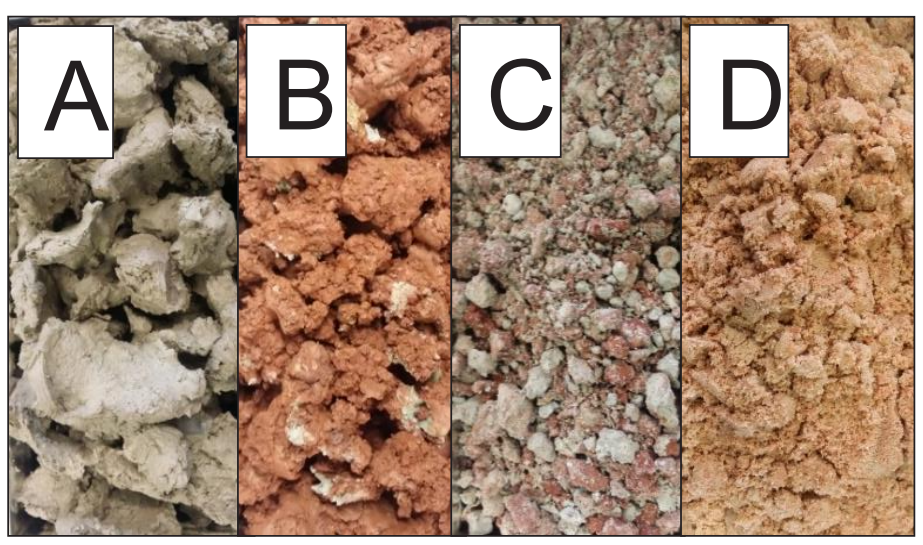

Figure 2. A - Grey clay; B - Red clay; C - Composite clay; D - Sand.

As surfactant used for sample making is Triton X-100 from sigma Aldrich. Triton X-100 or polyethylene glycol tert-octylphenyl ether is anionic surfactant (Figure 3).<smiles>CCOCCOc1ccc(C(C)(C)CC(C)(C)C)cc1</smiles>

Figure 3. Structural formula of Triton X-100 [13].

Contact angle was measured using Triton X$100 /$ water contact angle and drop test on glass plate. Contact angle was measured automatically for 30 seconds recording readings. Test was done three times for each concentration. The water contact angle measurements were made by the drop method by an optical tensiometer Theta Attension (Finland).

Surface tension was measured by plate method. Measurements were made by Kruss $100 \mathrm{M}$ tensiometer. Surface tension was detected at $25^{\circ} \mathrm{C}$ with Plate method (20x10x0,2 mm). Depth of emersion of plate $1 \mathrm{~mm}$.

Granulometry was made by the pipette method. Raw material was dried at $105^{\circ} \mathrm{C}$ sieved till $<2 \mathrm{~mm}$ fraction. Oxidation of organic matter was done by hydrogen peroxide $\left(\mathrm{H}_{2} \mathrm{O}_{2}\right)$. Samples were dipped in distilled water whit dispersant $\left(\mathrm{Na}_{2} \mathrm{PO}_{3}\right)+\mathrm{Na}_{2} \mathrm{CO}_{3}$ for $24 \mathrm{~h}$. The aim of pre-treatment is to promote dispersion of the primary particles, and to get more accurate clay size particle amount in the sample. Suspension was wet sieved on 63 $\mu \mathrm{m}$ sieve. Fraction $>63 \mu \mathrm{m}$ that left on sieve was dried at $105^{\circ} \mathrm{C}$ and dry sieved. Fraction that passed $63 \mu \mathrm{m}$ sieve was analysed by sedimentation method taking samples at appropriate intervals [14].

Cracks were analysed using digital camera (Moticam 2000) and software Motic Images Plus 2.0. 


\section{Results}

\subsection{Contact angle measurements and surface tension}

In table 1 is shown contact angle measurements between glass plate and different concentration droplet of Triton $\mathrm{X}-100$. Wettability increases or contact angle decreases by increasing concentration of Triton X-100. Using Triton X-100 concentration of $0,00001 \%$ contact angle is even higher than for water. Which means that to improve wettability lowest concentration that can be used is $0,0001 \%$.

Surface tension values are shown in table 1 . It can be concluded surface tension increases if surfactant concentration is decreased.

Table 1. Results of contact angle and surface tension measurements of different Triton X-100 concentrations.

\begin{tabular}{|l|l|l|l|l|l|l|}
\hline $\begin{array}{l}\text { Triton X-100 } \\
\text { molar } \\
\text { concentration }\end{array}$ & $\mathrm{H}_{2} \mathrm{O}$ & 0,1 & 0,01 & 0,001 & 0,0001 & 0,00001 \\
\hline $\begin{array}{l}\text { Contact } \\
\text { angle }^{\circ}\end{array}$ & 20,9 & 12,9 & 13,8 & 16,7 & 17,3 & 26,2 \\
\hline $\begin{array}{l}\text { Surface } \\
\text { tension } \\
\mathrm{mN} / \mathrm{m}\end{array}$ & 71,8 & 31,9 & 31,8 & 31,6 & 37,6 & 55,7 \\
\hline
\end{tabular}

In Figure 4 are shown $\mathrm{CMC}$ determination. $\mathrm{CMC}$ can be determinate easily at $0,001 \%$. Below this concentration surface tension values changes very slowly are almost constant, but above CMC surface tension changes very fast. All samples are made with surfactant concentration above $\mathrm{CMC}$ to achieve improvement of moisture transport and less cracking during drying.

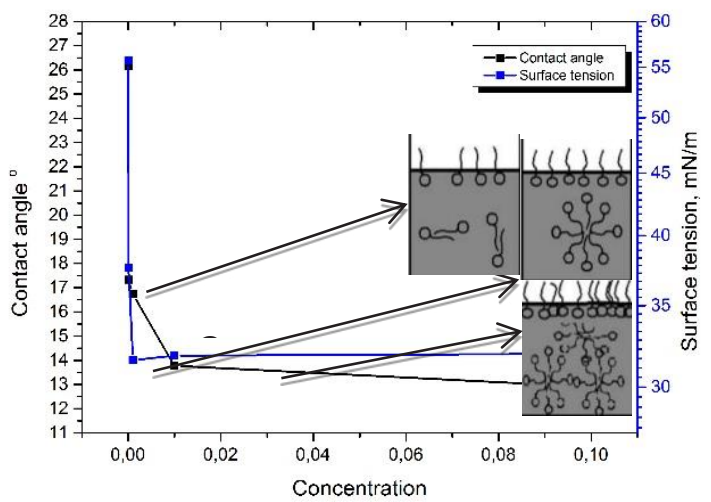

Figure 4. Contact angle and surface tension dependence on concentration. $\mathrm{CMC}$ detection.

\subsection{Granulometry}

Granulometry was carried out using pipette method for all samples. Granulometry in all three samples, VAV0,01 with Triton X-100 concentration $0,01 \%$, VAV0,001 with Triton X-100 concentration $0,001 \%$ and VAV0,0001 with Triton X-100 concentration $0,0001 \%$, are almost the same. Sand size particles $>20 \mu \mathrm{m}$ are about $45 \%$, silt size particles $2-20 \mu \mathrm{m}$ are about $25 \%$ and clay size particles are about $30 \%$. Due to Figure 4 B), sample granulometry is right in place for perforated bricks, but as we have extruded full bricks more sand size particles should be added to improve workability and drainage paths.
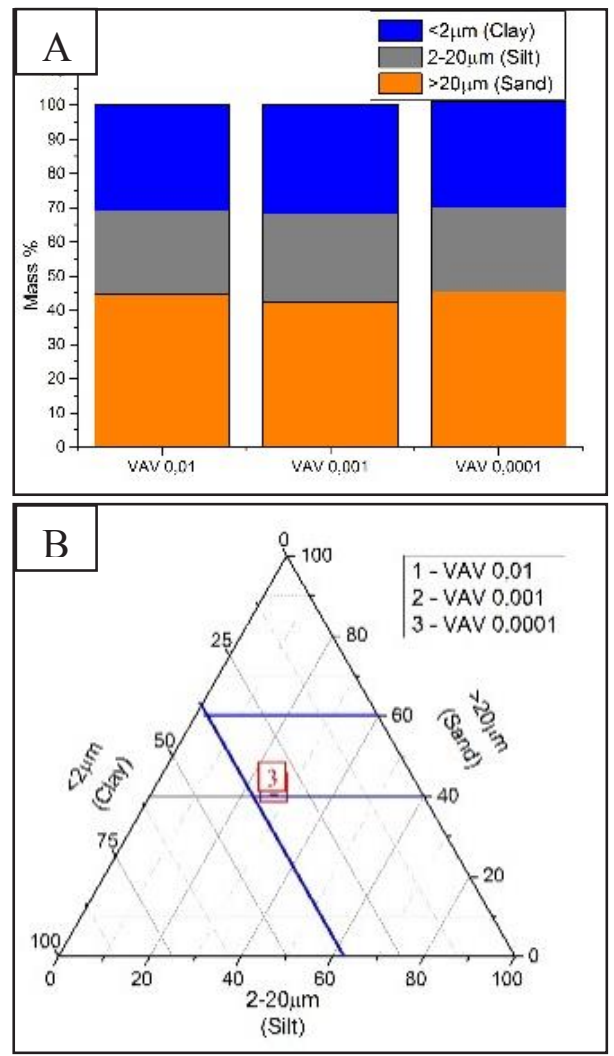

Figure 5. A) Particle size distribution of VAV0,01 with Triton X-100 concentration $0,01 \%$, VAV0,001 with Triton X-100 concentration $0,001 \%$ and VAV0,0001 with Triton X-100 concentration $0,0001 \%$; B) VAV0,01, VAV0,001, VAV0,0001 location in Winklers diagram.

\subsection{Crack amount}

In table 2 is shown crack amount as average crack area of dried sample. In different publications are different opinions of results below or above CMC [1]. The best quality of the dried samples was achieved for clay saturated with water containing rather low concentration of surfactants. But R. Guéganet. al. [10] have come to conclusions that nonionic surfactant (C10E3) concentration range above $\mathrm{CMC}$ or near $\mathrm{CMC}$, gives the maximum adsorption to montmorillonite clay surface.

Our suggested drying mechanisms with surfactants are shown in Figure 6. Hydrophilic head of surfactant molecule adsorbs to clay structure and hydrophobic tail stays in pours. Water molecules during drying process are rolling through hydrophobic tails and does not adsorb to other particles or do not interact in structure. This mechanism helps water molecules to move easier from brick centre to surface. 


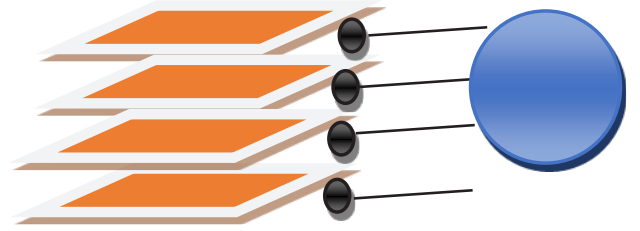

Figure 6. Drying mechanism in clay when surfactant is used.

\subsection{Evaluation of crack}

Cracks were measured after drying. Average crack area on sample area was measured. Results are shown in table 2. Better results are achieved if higher Triton X-100 concentration is used and it is closer to CMC.

Table 2. Crack amount of samples with different Triton X-100 concentrations.

\begin{tabular}{|l|l|l|l|}
\hline $\begin{array}{l}\text { Triton X-100 } \\
\text { molar } \\
\text { concentration }\end{array}$ & 0,01 & 0,001 & 0,0001 \\
\hline $\begin{array}{l}\text { Crack } \\
\text { amount, \% }\end{array}$ & 0,07 & 0,09 & 0,10 \\
\hline
\end{tabular}

In Figure 7 are shown sample crack measurements. Crack in samples are mostly concentrated in the middle and they are round shaped. Round shaped racks come from extruder auger. This type of crack is combination of flow lamination and rotational movement of clay mass. Also hallow space created by auger hub in the canter of emerging clay mass which have not entirely joined as a result of mass characteristics or geometry of pressure head and die [8]. So, these cracks are not from drying process and must be eliminated during forming process.

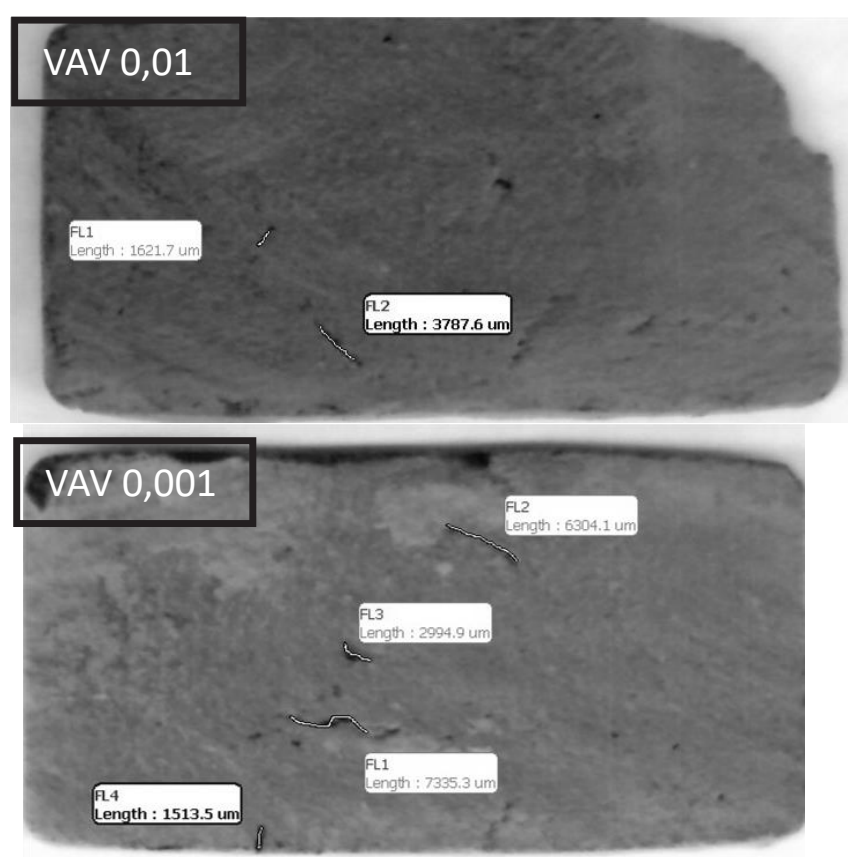

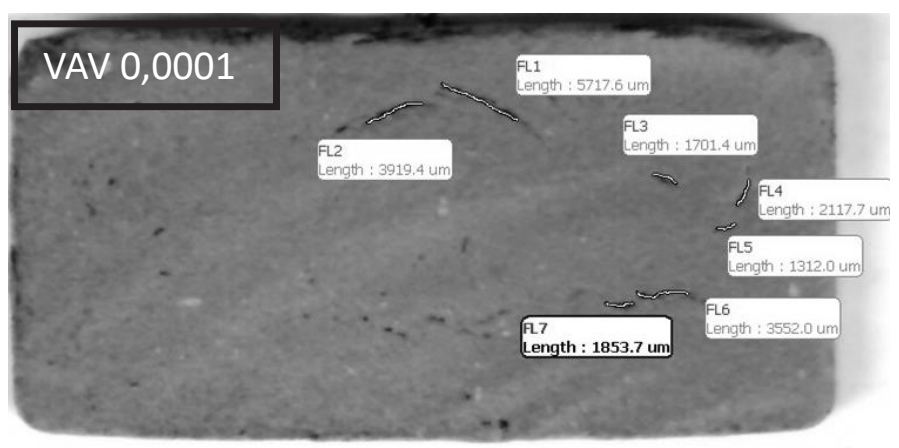

Figure 7. Sample crack measurements.

\section{Summary}

Due to information available in literature, surfactant addition to clay materials to develop drying performance is important theme, because cracking even in drying process is a large problem and needs to be controlled and eliminated. In our research crack development was measured after drying process for samples with added surfactant Triton X-100 near and below CMC. Crack amount decreases from $0,10 \%$ till $0,07 \%$ if Triton X-100 concentration is increased closer to CMC. Crack amount decreases, because surfactant molecules help water molecules to move easier from brick centre to surface reducing tension between dry brick surface and wet brick core. Our results show that to achieve lower crack amount during drying process surfactant must be added near CMC.

\section{References}

1. S. J. Kowalski and K. Kulczyński, Chem. Eng. Res. Des., vol. 91, no. 2, pp. 254-263, (2013).

2. N. Phonphuak, S. Kanyakam, and P. Chindaprasirt, J. Clean. Prod., vol. 112, pp. 3057-3062, (2016).

3. A brick drying room (2017).

4. Amido-amine based cationic Gemini surfactants for clay inhibition (2017)

5. N. Jones, S. D. N. Lourenço, and A. Paul, E3S Web Conf., vol. 9, p. 13006, (2016).

6. A. Yataganbaba and I. Kurtbaş, Renew. Sustain. Energy Rev., vol. 59, pp. 206-224, (2016).

7. O. A. Madyan, M. Fan, and Z. Huang, Appl. Clay Sci., vol. 141, pp. 64-71, (2017).

8. F. Händle, vol. 1542, no. 9. (2007).

9. M. J. Sánchez-Martín, M. C. Dorado, C. del Hoyo, and M. S. Rodríguez-Cruz, “J. Hazard. Mater., vol. 150 , no. 1 , pp. 115-123, (2008).

10. R. Guégan, Soft Matter, vol. 9, no. 45, p. 10913, ] (2013).

11. M. A. Migahed, M. M. Attya, S. M. Rashwan, M. Abd El-Raouf, and A. M. Al-Sabagh, Egypt. J. Pet., vol. 22, no. 1, pp. 149-160, (2013).

12. S. Guzlěna, G. Šakale, and S. Čertoks, Procedia Eng., vol. 172, pp. 333-337, (2017).

13. "TritonTM X-100 laboratory grade | Sigma-Aldrich." [Online].

Available: 
https://www.sigmaaldrich.com/catalog/product/sial/x 100 ?lang $=$ en\&region $=$ LV\&gclid $=$ CjwKCAjwdXaBRAEEiwAbwCi5gYxb2MaplVaEw77C0sU4fP 6cGdGiaO3QSilbhnGizuUU_3ViZ0TWBoC1VMQ AvD_BwE. [Accessed: 24-Jul-2018].
14. L. P. van Reeuwijk, Procedures for soil analysis, 6th ed. (2002). 